\title{
Marca-passo cardíaco definitivo em crianças com bradicardia pós-operatória: resultados tardios
}

\author{
Permanent cardiac pacing in children with postoperative bradycardia: long-term follow-up
}

Roberto COSTA, Kátia Regina da SILVA, Martino MARTINELLI FILHO, Wagner Tetsuji TAMAKI, Elizabeth Sartori CREVELARI, Luiz Felipe Pinho MOREIRA

\section{Resumo}

Objetivo: Avaliar a evolução tardia de crianças com marcapasso definitivo por bradicardia pós-operatória, e identificar fatores de risco para a mortalidade.

Método: De 1980 a 2004, 120 crianças receberam implante de marca-passo definitivo por bradicardia pós-operatória. $O$ intervalo médio entre a correção do defeito e o implante foi de 1,2 $\pm 2,8$ anos, com mediana de 21 dias. Bloqueio atrioventricular esteve presente em $94,2 \%$ dos pacientes. A via de acesso transvenosa $\mathbf{( 7 8 , 3 \% )}$ e marca-passos (MP) definitivos ventriculares $(\mathbf{7 9 , 2} \%)$ foram os mais utilizados. Empregou-se o método de Kaplan-Meier e o teste de LogRank para a análise de sobrevivência.

Resultados: Após 5,7 + 5,9 anos de seguimento (máximo= 22,5 anos), 97 pacientes estavam vivos e 11 haviam sido perdidos para o seguimento. As principais causas de morte foram insuficiência cardíaca (10), infecção não relacionada ao marca-passo (seis) e morte súbita (três). A expectativa de sobrevida aos cinco, 10 e 15 anos de seguimento mostrou, respectivamente, índices de 80,9 $\pm 4,1 \%, 75,4 \pm 5,5 \%$ e $67,2 \pm$ $7,4 \%$. A persistência de problemas hemodinâmicos após a correção (correções paliativas, uso de próteses valvares ou defeitos residuais) foi identificada como única variável preditora independente de risco para mortalidade, alterando significativamente as curvas de sobrevivência $(p=0,0123)$.

Conclusões: O implante de marca-passo em casos de bradicardia pós-operatória possibilitou boa expectativa de sobrevida. A realização de correções paliativas, assim como a presença de defeitos residuais ou de próteses valvares, foram os únicos fatores preditores de mau prognóstico para essas crianças.

Descritores: Estimulação cardíaca artificial. Bloqueio cardíaco. Pediatria. Procedimentos cirúrgicos cardíacos. Complicações pós-operatórias.

Trabalho realizado no Instituto do Coração (InCor) da Faculdade de Medicina da Universidade de São Paulo - Brasil.

Trabalho apresentado no $32^{\circ}$ Congresso da SBCCV, Vitória, em 30 de abril de 2005.

Endereço para correspondência:

Prof. Dr. Roberto Costa

Instituto do Coração (InCor) da Faculdade de Medicina da Universidade de São Paulo.

Av. Dr. Enéas de Carvalho Aguiar 44, $2^{\circ}$ andar. São Paulo, SP, Brasil. CEP 05403-000. Fone/fax: 55-11-3069-5284.

E-mail: rcosta@incor.usp.br 


\begin{abstract}
Objective: To evaluate the long-term outcomes of children submitted to permanent cardiac pacing due to postoperative bradycardia and to identify risk factors for mortality.

Methods: From 1980 to 2004, 120 children were submitted to permanent pacemaker (PM) implantation. Interval between the defect correction and PM implantation was $1.2 \pm 2.8$ years, on average (median $=21$ days). Atrioventricular blocks were present in $94.2 \%$ of patients. Transvenous leads $(\mathbf{7 8 . 3} \%)$ and ventricular pacemaker systems $\mathbf{( 7 9 . 2 \% )}$ were used in most of the cases. Risk factors were studied using the Cox proportional model. The Kaplan-Meier method and the LogRank test were used to analyze survival.

Results: After a mean of $\mathbf{5 . 7} \pm \mathbf{5 . 9}$ years $($ maximum $=\mathbf{2 2 . 5}$ years) of follow-up, 97 patients were alive and 11 were lost from the follow-up study. The main causes of death were terminal heart failure $(10)$, infection not related to
\end{abstract}

\section{INTRODUÇÃO}

O uso de marca-passo (MP) definitivo em crianças é raro, sendo a lesão cirúrgica do sistema de condução a principal causa de implante.

As bradiarritmias pós-operatórias permanentes têm sido relacionadas a alta morbidade, devido a arritmias ou comprometimento hemodinâmico. O bloqueio atrioventricular pós-operatório, em especial, está associado a elevada mortalidade, súbita e total, mesmo nos pacientes submetidos a correções consideradas completas [1].

A estimulação cardíaca artificial permanente reduz significantemente a mortalidade desses pacientes, sendo a evolução tardia adequada. A mortalidade está geralmente relacionada à doença de base e raramente a disfunções do sistema de estimulação.

O presente estudo foi desenvolvido com a finalidade de analisar a evolução tardia dos pacientes portadores de MP definitivo devido à bradicardia pós-operatória e identificar fatores de risco para a mortalidade.

\section{MÉTODO}

No período de novembro de 1980 a abril de 2004, 120 pacientes, com idade de até 18 anos e bradicardia pós-operatória persistente, foram submetidos a implante de MP cardíaco artificial permanente, no Instituto do Coração (InCor) da Faculdade de Medicina da Universidade de São Paulo (FMUSP).

No momento do primeiro implante de MP, a idade variou de 43 dias a 18 anos de vida, com média de 5,9 $\pm 5,0$ anos e mediana de 4,3 anos. O sexo era feminino em $65(54,2 \%)$ e masculino em $55(45,8 \%)$ crianças.

Bradicardia isolada com risco potencial de morte súbita implantation (six), and sudden death (three). The 5-, 10-, and 15 -year survival rates were $80.9 \pm 4.1 \%, 75.4 \pm 5.5 \%$ and 67.2 $\pm 7.4 \%$, respectively. The persistence of hemodynamic problems (palliative procedures, the use of valve prostheses or the presence of residual defects) was identified as the only independent risk predictors for mortality, with significant alterations in the survival curves $(p=0.0123)$.

Conclusion: The implant of permanent pacemakers in children provided good survival expectancy, mainly depending on the underlying disease and the type of the correction made. Palliative corrections, such as the presence of residual defects or valve prostheses were the only predictors of poor results in these children.

Descriptors: Cardiac pacing, artificial. Heart block. Pediatrics. Cardiac surgical procedures. Postoperative complications.

justificou o implante em $78(65,0 \%)$ pacientes, sinais ou sintomas de insuficiência cardíaca, em 22 (18,4\%), síncopes, em $10(8,3 \%)$ e pré-síncopes ou tonturas, em $10(8,3 \%)$.

Bloqueio atrioventricular total persistente (BAVT) foi o principal achado eletrocardiográfico $(81,7 \%)$. BAVT intermitente $(7,5 \%)$, bloqueio atrioventricular do $2^{\circ}$ grau do tipo II $(5,0 \%)$ e as diferentes formas da doença do nó sinusal $(5,8 \%)$ estiveram presentes nos demais pacientes.

O tempo médio transcorrido entre a correção do defeito que causou a lesão do sistema de condução e o implante de MP foi de 1,2 $\pm 2,8$ anos, com mediana de 21 dias. Apenas três pacientes foram submetidos ao implante de MP concomitantemente à correção do defeito que causou a bradicardia. O implante de MP foi realizado até o $14^{\circ}$ dia após a correção do defeito em $27,5 \%$ das crianças, do $15^{\circ}$ ao $30^{\circ}$ dia em $30,0 \%$, de um a seis meses em $18,3 \%$, de seis a 12 meses em 3,3\% e após um ano da correção em 18,3\%.

A correção de defeito submembranoso do septo interventricular foi o procedimento mais freqüentemente correlacionado ao traumatismo do sistema de condução elétrica do coração, de forma isolada em $15(12,4 \%)$ ou associada a outros defeitos em $23(19,0 \%)$ pacientes. A correção de defeito do septo atrioventricular foi realizada em $18(14,9 \%)$ e da tetralogia de Fallot em $15(12,4 \%)$ pacientes. Anomalia de Ebstein esteve presente em cinco $(4,1 \%)$ e outras valvopatias também em cinco $(4,1 \%)$ pacientes. Cardiopatias complexas foram corrigidas em 25 $(20,7 \%)$ crianças, com isomerismo atrial esquerdo em $6,6 \%$.

A correção dos defeitos cardíacos congênitos foi considerada completa e sem defeitos residuais ou próteses valvares em 74 $(61,7 \%)$ pacientes. Defeitos residuais em correções consideradas completas foram detectados em 12 (10,0\%) pacientes. Próteses valvares em correções completas foram utilizadas em $23(19,2)$ 
pacientes, em sua posição anatômica ou em enxertos tubulares valvulados. Correções paliativas foram realizadas em $11(9,1 \%)$ casos. Uma criança submetida à correção paliativa também recebeu prótese valvar.

Problemas não-cardíacos estiveram presentes em 11 crianças: síndrome de Down (nove); síndrome de Noonan (uma) e epilepsia (uma).

\section{Vias de acesso utilizadas}

No primeiro procedimento para implante de MP, 95 $(79,2 \%)$ pacientes foram submetidos à colocação de sistema ventricular, $18(15,0 \%)$ receberam MP atrioventricular, quatro $(3,3 \%)$ sistemas multi-sítio e três $(2,5 \%)$, atriais puros.

A via de acesso transvenosa foi utilizada em 94 (78,3\%) crianças, através de veia femoral $(57,5 \%)$, subclávia $(19,2 \%)$ ou cefálica $(1,6 \%)$. Implantes epimiocárdicos foram realizados em $26(21,7 \%)$ crianças, através de abordagem transtorácica $(15,8 \%)$ ou subxifóide $(5,9 \%)$.

O gerador de pulsos foi implantado na parede abdominal em 91 (75,8\%) pacientes e na parede torácica em 29 (24,2\%), seja na região infraclavicular ou submamária.

\section{Fatores de risco estudados}

Analisou-se a influência dos fatores considerados de risco para a mortalidade: a idade do paciente no momento da correção cirúrgica e do implante do MP, o sexo, a persistência de problemas hemodinâmicos após a correção (correções paliativas, uso de próteses valvares ou defeitos residuais), o intervalo de tempo entre a correção do defeito e o implante do MP, os achados eletrocardiográficos no momento do implante e a via de acesso utilizada para o implante do MP.

\section{Análise estatística}

A expectativa cumulativa da sobrevivência em relação ao tempo foi determinada pelo método não-paramétrico de Kaplan-Meier. Pacientes submetidos a transplante cardíaco ou remoção do MP tiveram sua evolução censurada no momento desses procedimentos. Um paciente submetido à remoção do MP e novo implante, cinco anos depois, teve sua evolução incluída duas vezes nas curvas de sobrevivência. A análise exploratória dos fatores de risco foi realizada pelo modelo proporcional de Cox. As diferenças entre a freqüência de eventos no tempo de acordo com as variáveis identificadas foram comparadas pelo Teste de Log-Rank.

Todos os dados foram analisados pelo software Statistical Package for Social Sciences (SPSS), considerandose como significantes os valores de $p<0,05$.

\section{RESULTADOS}

Os pacientes foram acompanhados, em média, por 5,7 \pm 6,0 anos, com variação de quatro dias a 22,5 anos, compreendendo 679,1 pacientes-anos de seguimento. Onze pacientes foram perdidos para o seguimento, no caso mais precoce logo após o implante de MP, e, no mais tardio, após 12,5 anos de seguimento (média $=2,5 \pm 1,0$ ).

Reoperações para a manutenção do sistema de estimulação cardíaca artificial foram realizadas em 82 oportunidades, devido a: exaustão do gerador de pulsos $(51,2 \%)$; crescimento da criança $(15,8 \%)$; problemas relacionados ao eletrodo $(15,9 \%)$ e infecção $(4,9 \%)$. Mudança do modo de estimulação, devido a insuficiência cardíaca, foi necessária em seis $(8,5 \%)$ pacientes: para o modo atrioventricular em três, para átrio-biventricular em três e para átrio-bifocal no ventrículo esquerdo em um paciente. Outras causas menos freqüentes indicaram reoperação em 3,7\% dos pacientes.

Após o implante inicial do MP definitivo, foram realizadas reoperações para correção de defeitos residuais em 20 pacientes: 13 correções de defeitos residuais, 11 substituições valvares e dois transplantes cardíacos.

A recuperação da condução atrioventricular ocorreu somente em uma criança, sendo possível, nesse caso, a interrupção do uso do MP cinco meses após o implante, durante reoperação para fechamento de comunicação interventricular residual e re-ampliacão de via de saída de ventrículo direito. Cinco anos depois, entretanto, essa paciente foi novamente submetida a implante de MP por bloqueio atrioventricular total intermitente.

Vinte e três $(19,2 \%)$ pacientes morreram durante o seguimento. O óbito mais precoce ocorreu 14 dias após o implante de MP e o mais tardio, 13,5 anos (média = 2,8 $\pm 3,9$ anos). As causas dos óbitos foram: insuficiência cardíaca terminal (10), infecção não relacionada ao MP (seis), morte súbita (três) e tromboembolismo pulmonar (um). Em três pacientes, a causa do óbito não foi determinada.

A expectativa de sobrevida aos cinco, 10 e 15 anos de seguimento mostrou, respectivamente, índices de 80,9 \pm $4,1 \%, 75,4 \pm 5,5 \%$ e $67,2 \pm 7,4 \%$ (Figura 1 ).

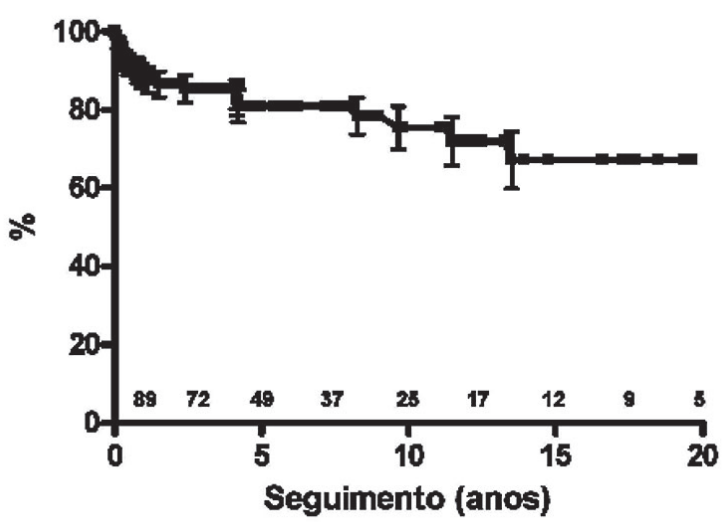

Fig. 1 - Análise, pelo método de Kaplan-Meier, da expectativa de sobrevida após o implante de marca-passo 
A análise exploratória dos fatores de risco, realizada pelo modelo proporcional de Cox, identificou persistência de problemas hemodinâmicos após a correção (correções paliativas, uso de próteses valvares ou defeitos residuais) como única variável preditora independente de risco para mortalidade. A Figura 2 mostra as curvas de sobrevivência de pacientes submetidos a correção total vs correção incompleta ou presença de próteses valvares, que apresentam diferenças significante $(p=0,0123)$ entre elas. Nos pacientes sem distúrbios hemodinâmicos, o risco relativo de morte de 0,36 ( 0,12 a 0,77 ; intervalo de confiança de $95 \%$ ) indicou uma redução no risco de morte de $64 \%$, mostrando que o risco de mortalidade dos pacientes submetidos a correções paliativas ou portadores de próteses valvares ou defeitos residuais foi 2,75 vezes maior que o dos pacientes submetidos a correções totais.

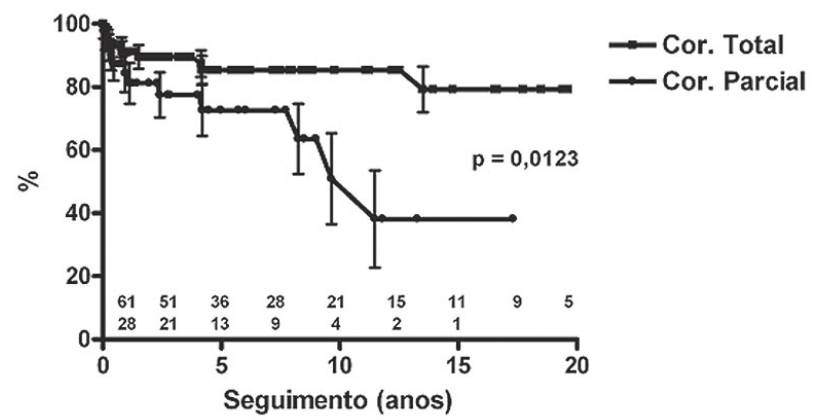

Fig. 2 - Avaliação da taxa de sobrevivência das crianças submetidas a correção total em comparação às submetidas a correções paliativas, com defeitos residuais ou com uso de próteses valvares. (Correção Parcial = paliativas, com defeitos residuais ou com próteses valvares)

\section{COMENTÁRIOS}

A necessidade do emprego da estimulação cardíaca artificial na população pediátrica é pouco comum, sendo, a sua principal indicação, a lesão cirúrgica do tecido de condução dos estímulos elétricos cardíacos durante cirurgia cardíaca. Nos casos em que ocorre o bloqueio atrioventricular irreversível, altos índices de mortalidade têm sido relatados.

$\mathrm{Na}$ literatura, a via de acesso epimiocárdica tem sido a preferida para implantes pediátricos, pelo acesso subxifóide ou transtorácico, particularmente em pacientes com menos do que $15 \mathrm{~kg}$. O acesso transvenoso, entretanto, tem apresentado melhores resultados tardios, com menores taxas de fratura de cabo-eletrodo e de perda de comando por aumento do limiar de estimulação.

Embora dados relacionados ao seguimento tardio de crianças com MP sejam pouco disponíveis, a estimulação cardíaca artificial reduz significativamente a mortalidade desses pacientes, de 60 a $80 \%$ em pacientes não tratados para cerca de $25 \%$ em pacientes estimulados [2,3].

Dados de seis estudos retrospectivos, que envolveram 796 crianças, mostraram a bradicardia pós-operatória como sendo a causa do implante de MP em 40 a $84 \%$ dos casos, sendo relatado o bloqueio atrioventricular congênito em 16 a $42 \%$ dos casos. Independentemente da causa da bradicardia, foi verificada a presença de defeitos cardíacos estruturais em 44 a $87 \%$ desses pacientes. Implantes epimiocárdicos foram realizados em 69 a 100\% dos procedimentos e a faixa etária dos pacientes variou de um dia a 21 anos, com média de 4,1 anos. Nesses estudos, a mortalidade pós-implante de MP variou de 4,1 a 22,9\% e a expectativa de cinco anos de sobrevida variou de 74 a $78 \%$, após seguimento médio de 2,4 a 11,9 anos [4-9].

Complicações da estimulação cardíaca artificial raramente causam ameaça à vida dessas crianças, tendo sido relatada mortalidade relacionada a problemas do MP em 2 a 4\% dos casos [5,10,11].

A incidência de implante de MP definitivo por bradicardia pós-operatória na literatura tem sido reportada entre 2 a $3 \%$ de todas as correções [12,13]. Weindling et al. [12] analisaram a incidência de todos os bloqueios atrioventriculares de $3^{\circ}$ grau, incluídos os reversíveis, em diferentes cardiopatias, encontrando incidência de $17 \%$ após a correção de defeito na via de saída do ventrículo esquerdo, $11 \%$ na correção da transposição das grandes artérias, $4 \%$ na correção de defeito do septo interventricular e de $3 \%$ na correção de tetralogia de Fallot, com necessidade de implante definitivo em aproximadamente $14 \%$ da população estudada.

O grande número de pacientes analisados no presente estudo, em se tratando de crianças com bradicardia pósoperatória, associado ao longo período de seguimento realizado, permitiram a obtenção de dados muito representativos, inclusive a análise dos fatores de risco para a mortalidade, aspecto nunca antes relatado na literatura para esse tipo de problema.

A expectativa de sobrevida total observada, de $80,9 \%$ aos cinco, $75,4 \%$ aos 10 e de $67,2 \%$ aos 15 anos de seguimento, foi compatível com a observada em crianças submetidas a correções semelhantes que não desenvolveram bradicardia ou necessidade de estimulação cardíaca artificial [14-24].

As causas de morte observadas no presente estudo, com predominância da insuficiência cardíaca terminal e de processos infecciosos não relacionados ao MP, corroboram relatos da literatura, que sugerem pequena importância de complicações da estimulação cardíaca artificial como causa de óbito.

A identificação da presença de problemas hemodinâmicos residuais como único fator de risco 
independente para a mortalidade, demonstrado no presente estudo, comprova a suposição previamente descrita por outros autores de que a mortalidade de crianças submetidas a implante de MP estaria relacionada principalmente à doença de base $[5,10,11]$.

Estes dados também estão em concordância com os encontrados na literatura para crianças submetidas a correções que não resultaram em bradicardia persistente: crianças submetidas a correções completas apresentam boa evolução tardia a despeito do uso de tubos extra-anatômicos não valvulados ou da persistência de discordância ventrículo-arterial. Por outro lado, as crianças submetidas a correções paliativas, com defeitos residuais após correções totais ou substitutos valvares, em posição anatômica ou em enxertos tubulares valvulados, têm apresentado pior evolução [14-25]. Spevak et al. [25] reportaram sobrevida $73 \%$ e $51 \%$ após um e cinco anos de seguimento, respectivamente, em crianças de até cinco anos de idade submetidas à substituição valvar.

\section{CONCLUSÕES}

O implante de marca-passo cardíaco artificial definitivo em crianças com bradicardia pós-operatória permitiu boa expectativa de sobrevida, dependendo, principalmente, da doença de base e do tipo de correção realizada. A realização de correções paliativas, assim como a presença de defeitos residuais ou de próteses valvares, foram os únicos fatores preditores de mau prognóstico para essas crianças.

\section{REFERÊNCIAS BIBLIOGRÁFICAS}

1. Bruckheimer E, Berul CI, Kopf GS, Hill SL, Warner KA, Kleinman CS et al. Late recovery of surgically-induced atrioventricular block in patients with congenital heart disease. J Interv Card Electrophysiol. 2002;6(2):191-5.

2. Lillehei CW, Sellers RD, Bonnabeau RC, Eliot RS. Chronic postsurgical complete heart block particular reference to prognosis, management, and a new P-wave pacemaker. J Thorac Cardiovasc Surg. 1963;46:436-56.

3. Fryda RJ, Kaplan S, Helmsworth JA. Postoperative complete heart block in children. Br Heart J. 1971;33(4):456-62.
4. Serwer GA, Mericle JM. Evaluation of pacemaker pulse generator and patient longevity in patients aged 1 day to 20 years. Am J Cardiol. 1987;59(8):824-7.

5. Kerstjens-Frederikse MW, Bink-Boelkens MT, de Jongste MJL, Homan van der Heide JN. Permanent cardiac pacing in children: morbidity and efficacy of follow-up. Int J Cardiol. 1991;33(6):207-14.

6. Rao V, Williams WG, Hamilton RH, Williams MG, Goldman BS, Gow RM. Trends in pediatric cardiac pacing. Can J Cardiol. 1995;11(11):993-9.

7. Sachweh JS, Vazquez-Jimenez JF, Schöndube FA, Daebritz SH, Dorge H, Muhler EG et al. Twenty years experience with pediatric pacing: epicardial and transvenous stimulation. Eur J Cardiothorac Surg. 2000;17(4):455-61.

8. Cohen MI, Bush DM, Vetter VL, Tanel RE, Wieand TS, Gaynor JW et al. Permanent epicardial pacing in pediatric patients: seventeen years of experience and 1200 outpatient visits. Circulation. 2001;103(21):2585-90.

9. Thomson JD, Blackburn ME, Van Doorn C, Nicholls A, Watterson KG. Pacing activity, patient and lead survival over 20 years of permanent epicardial pacing in children. Ann Thorac Surg. 2004;77(4):1366-70.

10. Esperer HD, Singer H, Riede FT, Blum U, Mahmoud FO, Weniger J. Permanent epicardial and transvenous single- and dual chamber pacing in children. Thorac Cardiovasc Surg. 1993;41(1):21-7.

11. Young D. Permanent pacemaker implantation in children: current status and future considerations. Pacing Clin Electrophysiol. 1981;4(1):61-7.

12. Weindling SN, Saul JP, Gamble WJ, Mayer JE, Wessel D, Walsh EP. Duration of complete atrioventricular block after congenital heart disease surgery. Am J Cardiol. 1998;82(4):525-7.

13. Bonatti V, Agnetti A, Squarcia U. Early and late postoperative complete heart block in pediatric patients submitted to openheart surgery for congenital heart disease. Pediatr Med Chir. 1998;20(3):181-6.

14. Clapp SK, Perry BL, Farooki ZQ, Jackson WL, Karpawich PP, Hakimi M et al. Surgical and medical results of complete atrioventricular canal: a ten-year review. Am J Cardiol. 1987;59(5):454-8.

15. Driscoll DJ, Offord KP, Feldt RH, Schaff HV, Puga FJ, Danielson GK. Five- to fifteen-year follow-up after Fontan operation. Circulation. 1992;85(2):469-96.

16. Duster MC, Bink-Boelkens MT, Wampler D, Gillette PC, McNamara DG, Cooley DA. Long-term follow-up of dysrhythmias following the Mustard procedure. Am Heart J. 1985;109(6):1323-6. 
17. Helbing WA, Hansen B, Ottenkamp J, Rohmer J, Chin JG, Brom AG et al. Long-term results of atrial correction for transposition of the great arteries: comparison of Mustard and Senning operations. J Thorac Cardiovasc Surg. 1994;1089(2):363-72.

18. Kyger ER 3rd, Frazier OH, Cooley DA, Gillette PC, Reul GJ Jr, Sandiford FM et al. Sinus venosus atrial septal defect: early and late results following closure in 109 patients. Ann Thorac Surg. 1978;25(1):44-50.

19. Manning PB, Mayer JE Jr, Sanders SP, Coleman EA, Jonas RA, Keane JF et al. Unique features and prognosis of primum ASD presenting in the first year of life. Circulation. 1994;90(5 Pt 2):II30-5.

20. Metcalfe J, Somerville J. Surgical repair of lesions associated with corrected transposition: late results. Br Heart J. 1983;50(5):476-82.
21. Ng R, Somerville J, Ross D. Ebstein's anomaly: late results of surgical correction. Eur J Cardiol. 1979;9(1):39-52.

22. Serraf A, Bruniaux J, Lacour-Gayet F, Sidi D, Kachaner J, Bouchart $\mathrm{F}$ et al. Anatomic correction of transposition of the great arteries with ventricular septal defect: experience with 118 cases. J Thorac Cardiovasc Surg. 1991;102(1):140-7.

23. Serraf A, Lacour-Gayet F, Bruniaux J, Ouaknine R, Losay J, Petit J et al. Surgical management of isolated multiple ventricular septal defects: logical approach in 130 cases. J Thorac Cardiovasc Surg 1992;103(3):437-43.

24. Turley K, Hanley FL, Verrier ED, Merrick SH, Ebert PA. The Mustard procedure in infants (less than 100 days of age): tenyear follow-up. J Thorac Cardiovasc Surg. 1988; 96(6):849-53.

25. Spevak PJ, Freed MD, Castaneda AR, Norwood WI, Pollack $\mathrm{P}$. Valve replacement in children less than 5 years of age. J Am Coll Cardiol. 1986;8(4):901-8. 